\title{
Avaliação do desempenho funcional em crianças com suspeita de transtorno do espectro autista
}

\author{
Evaluation of functional performance in children with \\ suspected autistic spectrum disorder
}

\section{Evaluación del rendimiento funcional en niños con sospecha de trastorno del espectro autista}

\author{
Aline Bernardes de Souza1, Luzia de Miranda Meurer², \\ Raquel Cymrot ${ }^{3}$
}

1.Fisioterapeuta. Doutora em Distúrbios de Desenvolvimento. Centro Universitário de Brusque - Unifebe. Brusque-SC, Brasil.

2.Psicóloga. Mestre em Educação. Centro Universitário de Brusque - Unifebe. Brusque-SC, Brasil.

3.Estatística. Mestre em Estatística. Universidade Presbiteriana Mackenzie. Brusque-SC, Brasil.

\begin{abstract}
Resumo
Introdução. $O$ transtorno do espectro autista (TEA) promove alterações no desenvolvimento infantil interferindo diretamente no seu desempenho funcional. Os seus sinais precoces podem ser observados entre o primeiro e o segundo ano de vida, mas tendem a ser identificados aos 5 anos de idade, no Brasil. Dados relacionados ao perfil e capacidade funcional podem auxiliar no reconhecimento das características autísticas do infante uma vez que as alterações comportamentais podem prejudicar o aprendizado e a performance nas AVDs promovendo uma maior necessidade de assistência para as suas execuções. Objetivo. Averiguar o desempenho funcional das crianças com risco para TEA e verificar se ele pode ser considerado uma possível ferramenta para o rastreamento do TEA. Método. Contou-se com a participação de 9 crianças do sexo masculino, que apresentavam risco para TEA segundo avaliação do Protea-R, com idades entre 2 anos e 6 meses a 3 anos e 5 meses de idade. A avaliação do desempenho funcional ocorreu pelo PEDI. Resultados. Os resultados encontrados demonstraram um baixo desempenho funcional nas crianças avaliadas, diferentes graus da necessidade de assistência nas AVDs e uma correlação positiva entre a função social e a dependência do cuidador. Conclusão. O PEDI apresenta um potencial contundente para o rastreamento precoce do TEA.
\end{abstract}

Unitermos. Autismo; Atividades Cotidianas; Criança; Programas de Rastreamento

\begin{abstract}
Introduction. Autistic Spectrum Disorders (ASD) promotes changes in child development, directly interfering with their functional performance. Its early signs can be seen between the first and second years of life, but they tend to be identified at 5 years of age, in Brazil. Data related to the profile and functional capacity can assist in the recognition of the infant's autistic characteristics since behavioral changes can impair learning and performance in ADLs, promoting a greater need for assistance for their executions. Objective. To investigate the functional performance of children at risk for ASD and to see if it can be considered a possible tool for ASD screening. Method. There was the participation of 9 male children, who were at risk for ASD according to the Protea-R assessment, aged 2 years and 6 months to 3 years and 5 months of age. Functional performance was assessed by PEDI. Results. The results found demonstrated a low functional performance in the children evaluated, different degrees of the need for assistance in the ADLs and a positive correlation between social function and dependence on the caregiver. Conclusion. PEDI has strong potential for the early screening of ASD.
\end{abstract}

Keywords. Autism; Daily Activities; Children; Tracking Programs 


\section{Resumen}

Introducción. El trastorno del espectro autista (TEA) promueve cambios en el desarrollo infantil, interfiriendo directamente en su desempeño funcional. Sus primeros signos se pueden ver entre el primer y segundo año de vida, pero suelen identificarse a los 5 años, en Brasil. Los datos relacionados con el perfil y la capacidad funcional pueden ayudar en el reconocimiento de las características autistas del bebé, ya que los cambios de comportamiento pueden afectar el aprendizaje y el desempeño en las AVD, promoviendo una mayor necesidad de asistencia para sus ejecuciones. Objetivo. Investigar el desempeño funcional de los niños en riesgo de TEA y verificar si puede considerarse una posible herramienta para el cribado de TEA. Método. Se contó con la participación de 9 niños varones, con riesgo de TEA según la evaluación Protea-R, con edades comprendidas entre 2 años y 6 meses a 3 años y 5 meses de edad. El desempeño funcional fue evaluado por PEDI. Resultados. Los resultados encontrados demostraron un bajo desempeño funcional en los niños evaluados, diferentes grados de necesidad de asistencia en las AVD y una correlación positiva entre la función social y la dependencia del cuidador. Conclusión. Se concluye, por lo tanto, que PEDI tiene un gran potencial para la detección temprana de TEA.

Palabras clave. Autismo; Actividades diarias; Niño; Programas de seguimiento

Trabalho realizado no Centro Universitário de Brusque - Unifebe. Brusque-SC, Brasil.

\section{INTRODUÇÃO}

O Transtorno do Espectro Autista (TEA) é classificado com um transtorno do neurodesenvolvimento que promove alterações no desenvolvimento infantil e que interfere diretamente no desempenho de suas atividades de vida diária (AVDs). Ele se expressa pelo comprometimento na comunicação e interação social da criança, nos mais variados contextos e, na presença de comportamentos restritos e repetitivos $^{1}$.

Alterações clínicas como epilepsia, disfunções gastrointestinais, distúrbios do sono, déficits intelectuais, comprometimentos psiquiátricos e motores podem estar associados ao TEA e apresentar diferentes variações de severidade pela intensidade da manifestação de seus sinais e sintomas ${ }^{1}$. 
Tarefas corriqueiras como fazer uso do toalete, vestirse, comunicar-se e desenvolver-se socialmente são alguns exemplos de atividades cotidianas que demonstram as alterações comportamentais do TEA e seu baixo desempenho funcional ${ }^{2,3}$.

Os sinais precoces do TEA podem ser notados entre 0 primeiro e segundo ano de vida da criança quando esta não consegue manter o contato visual com o outro, quando é apática ou extremamente inquieta e quando possui dificuldades de interação com os demais a sua volta. Porém, comumente no Brasil, a sua identificação tem ocorrido por volta dos 5 anos de idade da criança, quando esta inicia a sua vida educacional. Este fato se deve a inúmeros fatores como a variabilidade dos comportamentos motores, sensoriais e cognitivos do TEA e as limitações de acesso aos instrumentos avaliativos ${ }^{1,4}$.

Esta morosidade na identificação do transtorno tem incentivado profissionais brasileiros a desenvolverem novos instrumentos que avaliam a presença de características autísticas em indivíduos com suspeita de TEA como o Sistema PROTEA - R de Avaliação do Transtorno do Espectro Autista (Protea-R). Este tende a auxiliar a conduta terapêutica bem como quantificar o risco para o TEA da criança ${ }^{5-7}$.

Dados relacionados ao perfil e capacidade funcional da criança também podem auxiliar no reconhecimento das características autísticas do infante uma vez que as alterações comportamentais podem prejudicar 0 
aprendizado e a performance nas AVDs promovendo uma maior necessidade de assistência para as suas execuções.

Portanto, o objetivo deste estudo foi averiguar desempenho funcional de crianças com risco para TEA e verificar se o mesmo pode ser considerado uma possível ferramenta para averiguação das características autísticas.

\section{MÉTODO}

\section{Amostra}

Para este estudo contou-se com a participação de 9 crianças, do sexo masculino, com idades entre 2 anos e 6 meses a 3 anos e 5 meses de idade, média de 33,4 meses de idade. Destas, 6 crianças apresentaram risco para TEA e 3, risco relativo para TEA, segundo a avaliação do Protea-R. Nenhum dos infantes apresentava comorbidades associadas ao TEA. As crianças com risco para TEA foram designadas de $\mathrm{N} 1, \mathrm{~N} 2, \mathrm{~N} 3, \mathrm{~N} 4, \mathrm{~N} 5$ e N6 e, as crianças com risco relativo para TEA de N7, N8 e N9.

Esta pesquisa foi aprovada pelo Comitê de Ética em Pesquisa sob o CAAE no 11842519.0.0000.5636 e seguiu todas as recomendações da Comissão Nacional de Ética em Pesquisa (Resolução 466/2012).

\section{Procedimento}

O Protea-R é um instrumento de rastreamento da presença de comportamentos característicos do TEA em infantes com idades entre 24 a 60 meses. Sua avaliação é composta por uma entrevista semiestruturada, com os 
cuidadores da criança, para a identificação de possíveis sinais autísticos e; pela investigação de comprometimentos em 17 comportamentos.

A avaliação destes comportamentos é feita mediante a realização de brincadeiras semiestruturadas em três cenários distintos sendo eles: iniciativa de atenção compartilhada (IAC), resposta de atenção compartilhada (RAC), imitação (IMI), engajamento social (ES), sorriso (SOR), busca e resposta ao contato físico-afetivo (CFA), busca de assistência $(B A)$, protesto e/ou retraimento à interação $(P / R)$, exploração dos brinquedos (EXB), forma de exploração (FEX), coordenação viso motora (CV), brincadeira funcional $(B F)$, brincadeira simbólica (BS), sequência da brincadeira simbólica (SBS), movimentos repetitivos das mãos (MRM), movimentos repetitivos de outras partes do corpo (MRC) e comportamentos auto lesivos (CA).

Da avaliação destes 17 comportamentos o instrumento utiliza-se apenas de 5 itens para avaliação final e codificação das pontuações que correspondem a IAC, RAC, IMI, BS e os Movimentos Repetitivos de outras partes do corpo (MR). Cada item recebe um escore que varia entre 0 a 3 de acordo com a qualidade do comportamento apresentada durante a avaliação. De modo geral, quanto maior a presença de alterações de comportamento, maior será a pontuação em cada item. Por fim, a codificação final do teste classifica 0 indivíduo com Risco para TEA, Risco Relativo para TEA ou Sem Risco para TEA? 
Posteriormente, todos os infantes foram avaliados quanto ao seu desempenho funcional pelo Inventário de Avaliação Pediátrica de Incapacidade (PEDI).

O PEDI é um instrumento que proporciona uma caracterização pormenorizada do desempenho funcional da criança, de 6 meses a 7 anos e meio de idade, um prognóstico do seu desempenho futuro e o registro das suas alterações longitudinais. Ele é composto por um questionário estruturado, dividido em três partes, que apresenta informações sobre o desempenho de habilidades da criança (Parte I), da sua independência funcional ou da quantidade de ajuda fornecida pelo seu cuidador (Parte II) e das modificações necessárias no ambiente domiciliar para a realização das suas atividades de vida diárias (Parte III).

A Parte I do teste correlaciona-se às tarefas do cotidiano e subdivide-se em três áreas: autocuidado (AC), composta por 73 itens, mobilidade (MOB), constituída por 59 atividades funcionais e função social (FS), por 65 tarefas funcionais. A área de AC mensura a capacidade da criança em realizar tarefas como: alimentar-se, higienizar-se, cuidar dos cabelos, vestir-se, fazer uso do toalete e ter controle de esfíncter. $O$ item de MOB avalia a capacidade do infante em realizar atividades como: transferir-se no banheiro, em cadeiras, no carro, no ônibus e na cama, locomover-se em ambientes internos e externos, carregar objetos e, subir e descer escadas. E a área de FS averigua as habilidades da criança em tarefas funcionais que envolvem a compreensão de palavras e sentenças, o uso funcional da comunicação, a 
resolução de problemas, a interação com objetos e outras pessoas, a orientação temporal, a realização de tarefas domésticas e a autoproteção.

A Parte II refere-se à avaliação do grau de independência funcional da criança em 20 tarefas cotidianas avaliadas na parte I. Estas dividem-se em 8 itens relacionados a tarefas de $A C, 7$ de $M O B$ e 5 de FS. Cada uma destas áreas fornece um escore bruto total que posteriormente é transformado num escore normativo.

A Parte III, avalia a frequência das modificações ambientais, numa escala nominal, em categorias: nenhuma modificação, modificação centrada na criança, modificação de reabilitação ou modificação extensiva ${ }^{8}$.

A sua administração foi realizada com os pais das crianças participantes em uma sala reservada pela instituição parceira do estudo.

Os Termos de Consentimento autorizando a realização da pesquisa foram assinados pelos pais e/ou responsáveis pelos infantes participantes e pela entidade colaboradora do estudo. A amostra foi selecionada de maneira aleatória e de acordo com a disponibilidade de participação no estudo.

\section{Análise Estatística}

A análise estatística dos dados ocorreu de forma descritiva e com a utilização do programa Minitab. Para que o indivíduo apresente um desempenho funcional típico no PEDI ele deve demonstrar um escore normativo entre 30 a 70. Os valores abaixo de 30 demonstrou um déficit funcional 
e os acima de 70 uma performance superior ao esperado para a sua faixa etária.

Em alguns momentos, para uma melhor averiguação estatística entre os resultados normativos coletados nos grupos e os dados da população com desenvolvimento típico, o resultado do desempenho funcional foi codificado numa classificação proposta pelas autoras. Os coeficientes de desempenho inferiores a 30 foram codificados para o valor 0 , os valores entre 30 a 40 receberam a codificação igual 1, os resultados entre 40 a 50 foram transformados para 2, os escores de 50 foram alterados para 3, os dados entre 50 a 60 receberam o valor de 4 , os coeficientes entre 60 a 70 foram alterados para 5 e os resultados acima de 70 a codificação de 6 . Em todas as análises de dados foram adotadas o nível de significância de $10 \%$ em virtude do número da amostra.

\section{RESULTADOS}

A pontuação da amostra nos 5 comportamentos críticos avaliados pelo Protea-R pode ser visualizada no Gráfico $1 \mathrm{e}$ os escores normativos mensurados pelo PEDI no Gráfico 2 .

Durante o processo de aplicação do PEDI não houve relatos da inclusão ou da necessidade de modificações ambientais (parte III) para a realização do desempenho funcional das crianças avaliadas. 
Gráfico 1. Pontuação da amostra nos 5 comportamentos críticos avaliados pelo Protea-R.

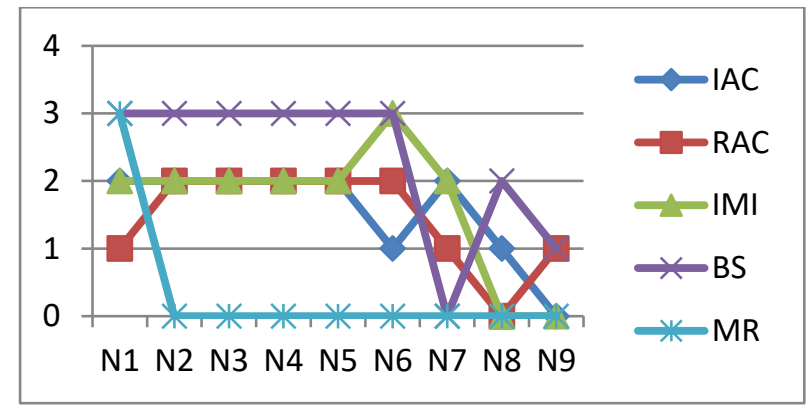

Gráfico 2. Escore normativo dos participantes nas áreas de Habilidades Funcionais (HF) e Assistência do Cuidador (Ass C).

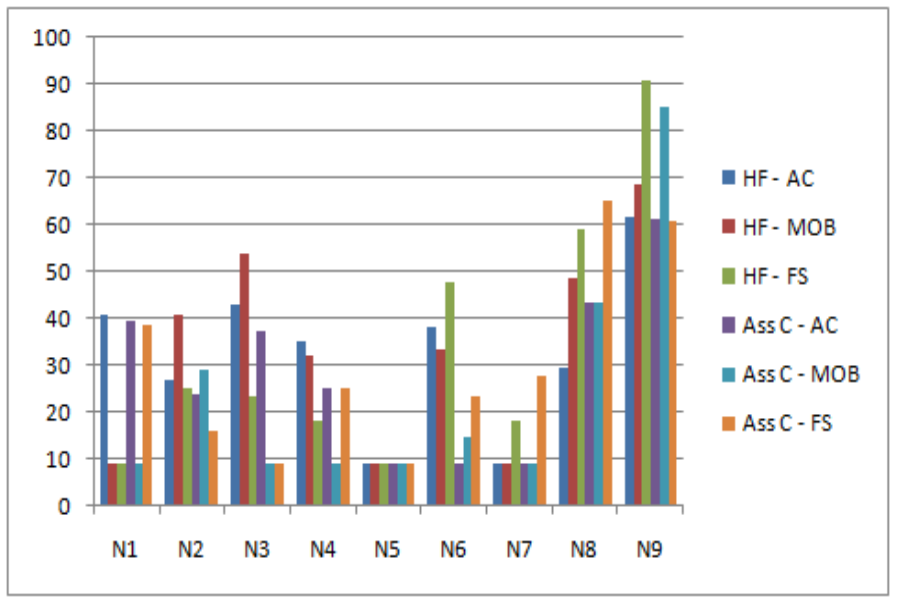

A comparação entre a média dos dados codificados das crianças com risco e risco relativo para TEA não apresentou diferenças significantes no teste de Mann-Whitney realizado: HF-AC, $p=0,892 ;$ HF-MOB, $p=0,416$; HF-FS, $p=0,203$; Ass C- $A C, p=0,416$; Ass C-MOB, $p=0,165$ e Ass C-FS, $p=0,199$.

Para a realização do teste qui-quadrado os dados codificados do PEDI foram agrupados em dois conjuntos, um de 0 a 2 e outro de 3 a 6 . Os itens HF - AC, p=0,33; HF MOB, $p=1$; Ass $C$ - AC, $p=1$ e Ass $C$ - MOB, $p=0,33$ não 
apresentaram dependência entre resultados altos e baixos das variáveis. Já as variáveis HF - FS, $p=0,08$ e Ass C - FS, $p=0,08$ demonstraram dependência entre escores codificados, altos e baixos, indicando que há mais crianças com valores de 0 a 2 no grupo com risco para TEA.

O teste de Wilcoxon demonstrou que as crianças aqui avaliadas apresentaram uma média inferior à população com desenvolvimento típico para as seguintes variáveis: $\mathrm{HF}$ - AC, $p=0,01 ; \mathrm{HF}-\mathrm{MOB}, \mathrm{p}=0,02 ; \mathrm{HF}-\mathrm{FS}, \mathrm{p}=0,04$; Ass C - AC, $p=0,02 ;$ Ass C - MOB, $p=0,02$ e Ass C - FS, $p=0,02$.

A Tabela 1 apresenta a correlação pareada de Spearman entre as variáveis do PEDI analisadas que se demonstraram positivas.

Já a correlação de Spearman entre as variáveis do Protea-R e PEDI foi positiva, apenas, para Ass C - MOB x BS, $\rho=-0,61$ e $p=0,07$.

Tabela 1. Correlação pareada de Spermann entre as variáveis da HF E ASS C do PEDI.

\begin{tabular}{|c|c|c|}
\hline Variáveis analisadas & Valor de rô & Valor de p \\
\hline HF - FS $\times$ Ass C - AC & $\rho=0,63$ & $p=0,06$ \\
\hline HF - FS $\times$ Ass C - MOB & $\rho=0,82$ & $p=0,00$ \\
\hline HF - FS $\times$ Ass C - FS & $\rho=0,70$ & $p=0,03$ \\
\hline Ass C - MOB $\times$ Ass C - AC & $\rho=0,87$ & $p=0,00$ \\
\hline Ass C - AC $\times$ Ass C - FS & $\rho=0,87$ & $p=0,00$ \\
\hline Ass C - MOB x Ass C - FS & $\rho=0,86$ & $\rho=0,86$ \\
\hline
\end{tabular}

\section{DISCUSSÃO}

Os resultados aqui encontrados demonstram que os indivíduos com risco para TEA apresentam uma variabilidade 
no desempenho funcional de suas AVDs e na necessidade de assistência do cuidador para com elas.

Mesmo não sendo possível identificar uma diferença significante entre os grupos avaliados, com risco e risco relativo, os escores normativos dos infantes com risco relativo para TEA apresentaram, de modo geral, uma performance funcional superior e uma maior independência funcional do que o grupo oposto. Este fato condiz com o grau de intensidade das características autísticas apresentadas pelas crianças na avaliação do Protea-R e que as difere em com risco ou com risco relativo para TEA.

O comportamento atípico dos indivíduos com TEA relacionados a interação, comunicação e reciprocidade social manifesta-se na dificuldade de contato visual e físico, na relação com o outro e no entendimento das regras sociais ${ }^{9}$. Estes comprometimentos foram notados na avaliação das atividades cotidianas de FS relacionadas a jogos interativos, brincadeiras com objetos, tarefas domésticas e resolução de problemas.

As habilidades de autocuidado e mobilidade do PEDI também se apresentaram prejudicadas ou com um escore abaixo da média em grande parte da amostra avaliada. Tais tarefas envolvem ações motoras de aprendizagem e desempenho, os quais não são descritos como critério diagnóstico para TEA, mas vem se demonstrando presente nesta população ${ }^{10,11}$.

A necessidade de assistência de um cuidador para a realização das suas atividades cotidianas demonstrou-se 
variada nos indivíduos aqui avaliados. Alguns apresentam dependência total, outros são semi-dependentes e outros independentes.

Essa variabilidade no grau de dependência relaciona-se ao nível de desempenho funcional e da intensidade das características autísticas dos infantes o que tende a proporcionar um alto índice de estresse e sobrecarga nos cuidadores $^{12,13}$.

Parece existir uma correlação entre a habilidade de FS e assistência fornecida pelo cuidador, nas suas 3 áreas funcionais, ou seja, quanto maior for o déficit da FS no sujeito maior será o seu grau de dependência.

Este dado ajusta-se às características do TEA que apresentam como comprometimento principal as habilidades sociocomunicativas. A dificuldade da criança em indicar a necessidade de fazer uso do banheiro, em interagir com pessoas próximas e compreender falas faz com que seja necessária uma assistência externa, constante, à criança, para a sua subsistência.

Os dados mostram, ainda, que existe uma correlação entre as áreas de assistência, ou seja, sempre que a criança necessitar de auxílio no desempenho de tarefas de uma área funcional necessitará da mesma assistência para desenvolvimento das atividades das outras duas áreas.

As variáveis de avaliação do PEDI não demonstraram, de modo geral, uma correlação efetiva com os itens finais do Protea-R. Tal fato, talvez ocorra, pelas diferentes propostas de avaliação de cada instrumento. O Protea-R investiga a 
presença de comportamentos específicos e o PEDI averigua o desempenho funcional da criança em diferentes contextos diários, o que envolve o desenvolvimento de diversas habilidades e comportamentos.

\section{CONCLUSÕES}

A pesquisa demonstra que as crianças com sinais autísticos apresentam uma alteração no seu desempenho funcional e que necessitam do auxílio de cuidador para a realização das suas AVDs.

Apesar de o estudo envolver um número restrito de participantes nota-se que o PEDI apresenta um potencial contundente para a identificação dos sinais característicos do TEA. Novos estudos sobre a temática, com uma população mais ampla, devem ser realizados para verificação aprofundado do assunto.

O conhecimento da performance funcional pode auxiliar, também, no desenvolvimento de condutas terapêuticas que promovam a funcionalidade da criança e minimizem a assistência no desempenho das atividades cotidianas, melhorando a qualidade de vida familiar.

\section{REFERÊNCIAS}

1.American PsychiatricAssociation (APA). Manual diagnóstico e estatístico de transtornos mentais: DSM-5. Porto Alegre: Artmed, 2014.

2.Silva WN, Rocha ANDC, Freitas FPM. Perfil de Crianças com Transtorno do Espectro Autista em Relação à Independência nas Atividades de Vida Diária. Rev Dial Persp Educ Esp 2018;5:71-84. https://doi.org/10.36311/2358-8845.2018.v5n2.06.p71

3. Matsukura TS, Menecheli LA. Famílias de Crianças Autistas: Demandas e Expectativas Referentes ao Cotidiano de Cuidados e o 
Tratamento. Cad Ter Ocup UFSCar 2011;19:137-52. http://www.cadernosdeterapiaocupacional.ufscar.br/index.php/cader nos/article/view/457/321

4.Bosa C. Atenção compartilhada e identificação precoce do Autismo. Psicol Refl Crít 2002;15:77-88. https://www.scielo.br/j/prc/a/P6p4y7ZqzhBVVfTPhXhB3Jc/?lang=pt\&f ormat $=$ pdf $\#:$ : : text $=$ Considerando $\% 20$ que $\% 20 a \% 20$ habilidade $\% 20 \mathrm{~d}$ e,e\%20fidedigno\%20indicador\%20precoce\%20de

5.Souza MS, Braga PP. O Inventário de Avaliação Pediátrica de Incapacidade e Suas Contribuições para Estudos Brasileiros. RevFundCare Online 2019;11:1368-75. http://dx.doi.org/10.9789/2175-5361.2019.v11i5.1368-1375.

6.American Occupational Therapy Association (AOTA). Estrutura da prática da Terapia Ocupacional: domínio \& processo - 3a ed. traduzida. Rev Ter Ocup USP 2015;26:1-49. https://doi.org/10.11606/issn.22386149.v26iespp1-49

7.Bosa CA, Salles JF. Sistema PROTEA-R de avaliação da suspeita de Transtorno do espectro Autista. São Paulo: Vetor, 2018.

8. Mancini MC. Inventário de Avaliação Pediátrica de Incapacidade (PEDI): manual da versão brasileira adaptada. Belo Horizonte: UFMG, 2005.

9.Swaiman K, Ashwal S, Ferriero D, Schor N, Finkel R, Gropman A, et al. Swaiman's Pediatric Neurology. In: Hirtz D, Wagner A, Filipek P, Sherr E (ed.). Autistic Spectrum Disorders. 6 ed. Edinburgh: Elsevier. 2018; p459-71.

10.Catelli CLRQ, D`antino MEF, Blascovi-Assis SM. Aspectos Motores em Indivíduos com Transtorno do Espectro Autista: Revisão de Literatura. CPDD 2016;16:56-65. https://www.mackenzie.br/fileadmin/ARQUIVOS/Public/6-posgraduacao/upm-higienopolis/mestradodoutorado/disturbios desenvolvimento/2016/cadernos/1/ASPECTOS MOTORES EM INDIVIDUOS COM TRANSTORNO DO ESPECTRO AU TISTA - REVISAO DE LITERATURA.pdf

11.Teixeira BM, Carvalho FT, Vieira JRL. Avaliação do Perfil Motor em Crianças de Teresina - PI com Transtorno do Espectro Autista (TEA). Rev Educ Esp 2019;32:71. https://doi.org/10.5902/1984686X33648 12.Tabaquim MLM, Vieira RGS, Razera APR, Ciasca SM. Autoeficácia de cuidadores de crianças com o transtorno do espectro autista. Rev Psicoped 2015;32:285-92. http://pepsic.bvsalud.org/pdf/psicoped/v32n99/02.pdf

13. Faro KCA, Santos RB, Bosa CA, Wagner A, Silva SSC. Autismo e mães com e sem estresse: análise da sobrecarga materna e do suporte familiar. Psico (Porto Alegre) 2019;50:e30080. https://doi.org/10.15448/1980-8623.2019.2.30080 